\title{
PRIORITIZED MULTI-COMMODITY FLOW MODEL AND ALGORITHM
}

\author{
DURGA PRASAD KHANAL ${ }^{1}$, URMILA PYAKUREL ${ }^{2}$, TANKA NATH DHAMALA ${ }^{3}$
}

\begin{abstract}
Prioritization is the process of deciding the relative importance or urgency of things or objects. It helps to focus on the objectives and goals in an efficient way. Prioritized flow problems are applicable for large scale disaster management problems. In this work, we introduce the priority based multi-commodity flow problem and develop a mathematical model. We also present a polynomial time algorithm to solve it.
\end{abstract}

Keywords: Network flow, Multi-commodity, commodity priority.

\section{INTRODUCTION}

Network flow models are widely used to solve real life problems since last sixty decades. Ford and Fulkerson [4] introduced the maximum static as well as dynamic flow problem in which maximum amount of flow is shifted from the source node to the sink node. To transship the flow with priority, Minieka [8] introduced the lexicographic maximum static flow problem and presented polynomial time algorithm to solve it. Megiddo [7] studied this problem in single source and multi sink network. Hamacher and Tufekci [5] presented evacuation problem as a lexicographic min cost flow problem and presented an algorithm based on time expanded network. They divided the building in to different prioritized zone and evacuate with successive higher priority zones as quickly as possible. The first polynomial time algorithm to solve lexicographic maximum dynamic flow and quickest transshipment problems are introduced by the same authors in [6]. Pyakurel and Dempe [9] introduced the prioritized maximum flow problem with contraflow approach and presented polynomial time algorithm with intermediate storage.

Multi-commodity flow problem concerns with the transshipment of more than one commodities from respective sources to corresponding sinks without violating capacity constraints on the arcs where as maximum multi-commodity flow problem deals with the transshipment of maximum number of objects. Fleischer and Skutella [3] generalized the dynamic flow problem introduced by Ford and Fulkerson [4] to the case of a multicommodity network. The static multi-commodity flow problem is solved in polynomialtime by using the ellipsoid or interior point method. For detail illustration in single and multi-commodity flows, we refer to $[1,2,3,10]$.

In this work, we introduce the model for the prioritized multi-commodity flow problem. After mathematical formulation of prioritized multi-commodity flow problem in Section 2, we present polynomial time algorithm to solve the problem in Section 3. We conclude it in Section 4.

\section{Mathematical Formulation of Flow Model}

The multi-commodity flow problem with priority order concerns with the transshipment of distinctly categorized objects from their respective sources to corresponding sinks without violating the capacity constraints through a given network topology. 
2.1. Notations. Let $\mathcal{N}=\left(V, A, K, u, d_{i}, S, D\right)$ be a network topology with node set $V$, arc set $A \subseteq V \times V$, and the set of commodities $K=\{1,2, \ldots, k\}$. The non-negative capacity function $u: A \rightarrow \mathcal{R}^{+}$limits the flow of commodities. We use $n$ and $m$ to denote the number of nodes and arcs in $\mathcal{N}$. The demands for each commodity $i \in K$, denoted by $d_{i}$, is routed through a unique source-sink pair $\left(s_{i}, t_{i}\right)$, where $s_{i} \in S \subset V$ and $t_{i} \in D \subset V$ and $S \cap D=\emptyset$. The flow function is defined by $x: A \rightarrow \mathcal{R}^{+}$, where $x_{e}^{i}$ represents the flow of commodity $i$ on $\operatorname{arc} e$.

2.2. Priority order. Prioritization reflects the importance of things or objects which may depend on its objective and goal. For example, in case of disasters, priority is given according to case sensitive like critically insured, minor insured, old aged people and pregnant women or with babies, normal people and shifted on respective destinations like well equipped hospitals, health centers, care centers and safe shelters, respectively. Thus, the problem becomes a multi-commodity flow problem with commodity priority, where commodities are transshipped from respective sources (collection centers $s_{i}$ ) to corresponding sinks (destinations $t_{i}$ ) without violating capacity constraints on the arcs.

We define the commodity priority ordering function $\mathbb{P}: K \rightarrow \mathcal{Z}^{+}$such that $\mathbb{P}(i) \prec$ $\mathbb{P}(i+1) \quad \forall i \in K$, where $\mathbb{P}(1)$ represents the commodity with first priority that is to be transshipped from $s_{1}$ to $t_{1}$ and so on. If more than one commodities are to be entering on an arc, then commodity of first priority $\mathbb{P}(1)$ enters at first, and if flow of first commodity is strictly less than arc capacity, then commodity of second priority $\mathbb{P}(2)$ is to be entered and so on with successive order.

The priority ordering in term of path flow is as follows. Let $\mathbf{P}_{i}$ be a set of paths of commodity $i$ (i.e. objects of priority group $i$ ). The bottleneck capacity of path $P \in \mathbf{P}_{i}$ is

$$
\nu_{P}^{i}=\min \left\{u_{e} \mid e \in P\right\} .
$$

The prioritized flow on bundle arc $e$ with priority order is

$$
x_{e}^{i}=\left\{\begin{array}{ll}
\nu_{P}^{1} & \text { if } i=1 \\
\min \left\{u_{e}-\sum_{m=1}^{i-1} x_{e}^{m}, \quad \nu_{P}^{i}\right\} & \text { if } i \geq 2
\end{array} \quad \forall P \in \mathbf{P}_{i} .\right.
$$

2.3. Prioritized flow model. A static multi-commodity flow $x$ for the given network $\mathcal{N}$ is the sum of all nonnegative static flows $x^{i}$ defined by the functions $x^{i}: A \rightarrow \mathcal{R}^{+}$for each commodity $i$ satisfying the prioritized flow function (2.1) and constraints (2.2 - 2.3).

$$
\begin{aligned}
\sum_{e \in A_{v}^{\text {out }}} x_{e}^{i}-\sum_{e \in A_{v}^{i n}} x_{e}^{i} & =\left\{\begin{array}{r}
d_{i} \text { if } v=s_{i} \\
-d_{i} \text { if } v=t_{i} \\
0 \text { otherwise }
\end{array} \quad \forall i \in K\right. \\
0 \leq x_{e} & =\sum_{i \in K} x_{e}^{i} \leq u_{e} \forall e \in A
\end{aligned}
$$

The sets $A_{v}^{i n}=\{(w, v) \mid w \in V\}$ and $A_{v}^{\text {out }}=\{(v, w) \mid w \in V\}$ denote the set of incoming arcs to node $v$ and outgoing arcs from node $v$, respectively, such that $A_{D}^{\text {out }}=\emptyset$ and $A_{S}^{i n}=\emptyset$. Third condition of the constraints in (2.2) are flow conservation constraints for each commodity at intermediate nodes and the net flow is presented in the remaining conditions. The constraints in (2.3) are bundle constraints bounded by arc capacities.

\section{Commodity PRIORITIZED MUlTi-COMmodity FLOW}

Prioritization is the sequentially deciding the importance of the objects. Minieka [8] has shown the existence of lexicographic flow in static network. For given set of sources $\left\{s_{1}, \ldots, s_{k}\right\}$, he constructed the set of chain of sources $S_{i}=\left\{s_{1}, \ldots, s_{i}\right\} \quad \forall i \in K$ and proved that the amount of flow leaving the sources $S_{i}$ is simultaneously maximum. Our model is prioritized multi-commodity flow model where each commodity is to be transshipped from respective source to corresponding sink. So prioritization of sources automatically prioritize the sink in same order. Together with this, our model also fixes 
the priority of commodities in each bundle arcs where, more than one commodities are entering on the same arc.

In this section, we introduce the multi-commodity flow problem with commodity priority and present an algorithm to solve it.

Problem 1. Let $\mathcal{N}=\left(V, A, K, u, d_{i}, S, D\right)$ be the given multi-commodity flow network with commodity priority order $\mathbb{P}(i) \prec \mathbb{P}(i+1) \quad \forall i \in K$. Then the prioritized multicommodity flow problem is to transship the net flow $d_{i}$ from $s_{i}$ to $t_{i}$ by using priority order without violating the capacity constraints on arcs.

To solve this problem, we first prioritize the commodities and set $S_{1}=s_{1}, S_{2}=$ $s_{1} \cup s_{2}, \ldots, S_{k}=\cup_{i=1}^{k} s_{i}$ so that $S_{1} \subseteq S_{2} \subseteq \cdots \subseteq S_{k} \subseteq S$. While sending flow on bundle arcs, priority is given as described in Section 2.2. We use lex-max flow algorithm of Minieka [8] to solve the problem in polynomial time. Here, we present an algorithm to solve the problem.

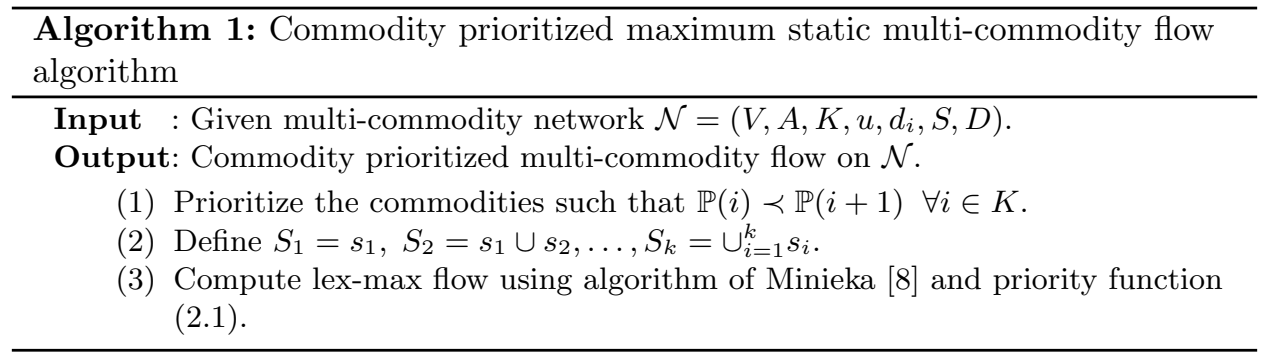

Theorem 3.1. Algorithm 1 solves commodity prioritized maximum static multi-commodity flow problem efficiently.

Proof. First we prove the feasibility of the theorem. Steps 1 and 2 are feasible which can be obtained in constant time. The priority function (2.1) is applied in each intermediate arcs within $O(m)$ times. As lex-max algorithm of Minieka [8] is polynomial time solvable, Step 3 is also feasible. Next, the optimality of algorithm is dominated by the optamilaty of Step 3 which provides optimal solution for each $S_{k}=\cup_{i=1}^{k} s_{i}$.

Example 1. Consider a multi-commodity network with three commodities that are to be transshipped from $s_{i}$ to $t_{i}$ for $i=1,2,3$. While sending flow on bundle arc $(v, w)$, first priority is given to commodity- 1 with bottleneck capacity 4 on the path $s_{1}-v-w-t_{1}$. The remaining capacity on the arc is $11-4=7$, so commodity- 2 is transshipped through path $s_{2}-v-w-t_{2}$ with flow of 5 units, because the minimum of remaining capacity of arc and bottleneck capacity of path is 5 . Similarly, we can send 2 units of flow on the path $s_{3}-v-w-t_{3}$ for commodity-3. Total amount of prioritized flow for commodity-1, commodity- 2 and commodity- 3 are 7,5 and 6 units, respectively.

\section{Conclusion}

Transmission of different type of commodities from respective sources to corresponding sinks with priority order and without violating capacity constraints is the prioritized multicommodity flow problem. In this work, we introduced prioritized multi-commodity flow problem and solved it by using lex-max flow of Minieka [8]. We reduce it to the single commodity flow problem by using priority ordering of each commodity. 


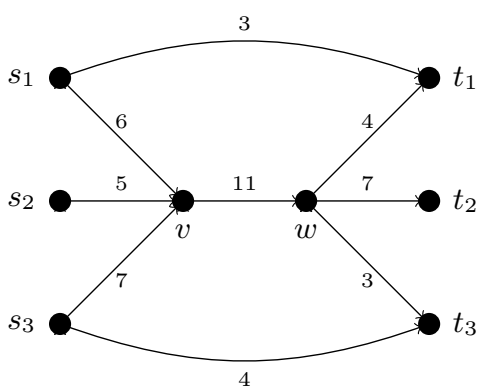

(a) arc capacity

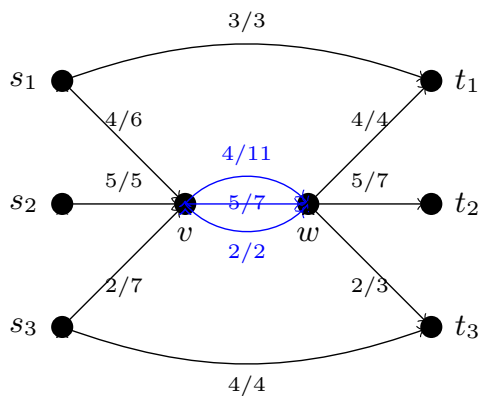

(b) flow/capacity

FIGURE 1. (a) multi-commodity flow network, (b) solution with priority order.

\section{REFERENCES}

1. Dhamala, T.N., Pyakurel, U. and Dempe, S. (2018). A critical survey on the network optimization algorithms for evacuation planning problems. International Journal of Operations Research, 15(3), 101-133.

2. Fleischer L. (2000). Approximating fractional multi-commodity flow independent of the number of commodities. SIAM Journal on Discrete Mathematics, 136, 505-520.

3. Fleischer, L. and Skutella, M. (2007). Quickest flows over time. SIAM Journal on Computing, 36(6), 1600-1630.

4. Ford, L.R. and Fulkerson D.R. (1962). Flows in Networks. Princeton University Press, Princeton, New jersey.

5. Hamacher, H.W. and Tufekci, S. (1987). On the use of lexicographic min cost flow in evacuation modeling. Naval Research Logistics, 34, 487-503.

6. Hoppe, B. and Tardos, E. (2000). The quickest transshipment problem. Mathematics of Operations Research, 25, 36-62.

7. Megiddo, N. (1974). Optimal flows in networks with multiple sources and sinks, Mathematical Programming, 7, 97-107.

8. Minieka, E. (1973). Maximal Lexicographic and Dynamic Network flows. Operations Research 21, 517-527

9. Pyakurel, U., Dempe, S. (2020). Network Flow with Intermediate Storage: Models and Algorithms. SN Operations Research Forum, DOI: 10.1007/s43069-020-00033-0.

10. Pyakurel, U., Gupta, S.P., Khanal D.P. and Dhamala, T.N. (2020). Efficient algorithms on multi-commodity flow over time problems with partial lane reversals. International Journal of Mathematics and Mathematical Sciences, accepted.

1 Tribhuvan University, Saraswati Multiple Campus, Nepal, ${ }^{2,3}$ Central Department of Mathematics,Tribhuvan University, Kathmandu, NePal

E-mail address: ${ }^{1}$ durgapsdkhanal@gmail.com, ${ }^{2}$ urmilapyakurel@gmail.com

E-mail address: ${ }^{3}$ amb.dhamala@daadindia.org 